\title{
Influence du repas sur l'activité enzymatique des différentes populations microbiennes du rumen
}

\author{
C Martin, B Michalet-Doreau \\ INRA, station de recherche sur la Nutrition des herbivores, unité de la Valeur alimentaire INRA, \\ Theix, 63122 Saint-Genès-Champanelle, France
}

\begin{abstract}
Summary - Influence of time after feeding on enzymatic activity in different microbial populations from rumen digesta. The activities of polysaccharidases and glycosidases were monitored in the liquid-associated bacterial (LAB) protozoal (LAP) and solid-associated bacterial fractions (SAB) separated from bovine rumen content collected $1 \mathrm{~h}$ before and $3 \mathrm{~h}$ after feeding. Enzyme activities were most active in the SAB and remained constant in the LAP. Enzymes involved in the degradation of soluble carbohydrates increased in the LAB $3 \mathrm{~h}$ post-feed period and at the end of the postprandial period ( $1 \mathrm{~h}$ before) in the SAB.
\end{abstract}

L'activité enzymatique des différentes populations microbiennes du rumen, bactéries et protozoaires associées à la phase liquide (LAB, LAP) et bactéries adhérentes à la phase solide (SAB), détermine la vitesse de dégradation des glucides alimentaires, et plus particulièrement des glucides pariétaux. Elle varie en fonction de I'heure de prélèvement par rapport au repas et du type de population microbienne considérée (Williams et al, 1989). Par ailleurs, la population de protozoaires (LAP) a rarement été dissociée de celle des bactéries ( $L A B$ ), or elle jouerait un rôle non négligeable dans la dégradation des constituants pariétaux (Czerkawski et al, 1988). Aussi, dans cette étude, nous avons voulu mettre en évidence l'effet du repas sur l'activité enzymatique respective de 3 populations microbiennes: LAB, LAP et $\mathrm{SAB}$.

Matériel et méthodes - Quatre vaches taries de race jersiaise, munies d'une canule du rumen, reçoivent en quantité limitée une ration constituée exclusivement de foin (16,5\% MAT) et distribuée en 1 repas/j à $8 \mathrm{~h}$. Un échantillon représentatif de contenu ruminal est prélevé $1 \mathrm{~h}$ avant le repas et $3 \mathrm{~h}$ après, puis filtré pour séparer la phase solide de la phase liquide. Après dilution, incubation et élimination des particules, le liquide est centrifugé ( $1000 \mathrm{~g}, 10 \mathrm{~min}, \mathrm{~T}^{\circ}$ ambiante). Le surnageant est centrifugé à nouveau (15000 g, $20 \mathrm{~min}, 4^{\circ} \mathrm{C}$ ) pour isoler les bactéries de la phase liquide (LAB), tandis que le culot de protozoaires est abondamment lavé puis filtré sur un tissu de $20 \mu$ (LAP). Dix grammes de phase solide sont lavés, broyés et battus au stomacher $(5 \mathrm{~min})$ pour détacher les bactéries adhérent aux particules (SAB). Chaque population est reprise dans $20 \mathrm{ml}$ de conservateur puis soniquée $(4 \times 30 \mathrm{~s})$. Le surnageant obtenu après centrifugation $\left(5000 \mathrm{~g}, 15 \mathrm{~min}, 4^{\circ} \mathrm{C}\right)$ est utilisé pour les mesures d'activités enzymatiques. L'ensemble de ces traitements se fait en anaérobiose.

L'activité des glycosidases et des polysaccharidases (voir liste dans le tableau I) est respectivement déterminée en dosant par spectrophotométrie la quantité de $p$-nitrophénol et la quantité de sucres réducteurs liberés à partir de différents substrats glucidiques (Williams et al, 1984). L'activité enzymatique de chaque préparation est rapportée à la quantité de protéines dosée, selon la technique de Bradford (1976). Les résultats ont été traités par une analyse de variance à 3 facteurs de variations, l'animal, le temps et le type de population microbienne, avec interaction entre la population et le temps (procédure GLM de SAS).

Résultats et discussion - Les SAB ont une activité enzymatique systématiquement supérieure à celle des populations de la phase liquide, l'activité des LAB étant 
proche de celle des LAP à l'exception de la $\beta$-D-galactosidase et $\alpha$-D-glucosidase. L'activité glucidique des LAP ne varie pas avant $(-1 h)$ ou après le repas $(+3 h)$ quel que soit le substrat considéré. $A u$ contraire, pour les $L A B$ et les $S A B$, l'ingestion de foin a un effet significatif sur les principales enzymes intervenant dans la dégradation des sucres simples. L'activité de la $\beta$-D-xylosidase, $\beta$-D-glucosidase et $\alpha$-D-glucosidase des LAB augmente $3 \mathrm{~h}$ après le repas, alors que celle des $\mathrm{SAB}$ ( $\beta$ $D$-xylosidase, $\alpha$-D-glucosidase) est plus active $1 \mathrm{~h}$ avant. Ces résultats sont en accord avec ceux obtenus par Williams et al (1984, 1989). L'acitivité des polysaccharidases est semblable au point $-1 \mathrm{~h}$ et $+3 \mathrm{~h}$ pour les 3 populations microbiennes.

II semblerait donc que, dans le processus de la dégradation des sucres simples, la population bactérienne de la phase li- quide intervienne dans les heures qui suivent le repas, alors que la population bactérienne de la phase solide est d'autant plus active que l'on s'éloigne du repas. $\mathrm{Ce}$ temps de latence serait nécessaire, d'une part, à la fixation des bactéries aux particules et, d'autre part, à la dégradation des polysaccharides en sucres simples. Les variations nycthémèrales de l'activité polysaccharidique des SAB (Williams et al, 1989) n'ont pu être mises en évidence avec les heures de prélèvement fixées pour cet essai.

Bradford M (1976) Anal Biochem 72, 248-254

Czerkawski JW, Cheng KJ (1988) The Rumen Microbial Ecosystem (Hobson PN, ed). Elsevier, London, 361-385

Williams AG, Strachan NH (1984) Curr Microbiol 10, 215-220

Williams AG, Withers SE, Strachan NH (1989) $J$ Appl Bacteriol 66, 15-26

Tableau I. Activité enzymatique spécifique ${ }^{1}$ des $L A B, L A P, S A B$ isolés à partir de la phase liquide et solide de contenu ruminal de 4 vaches.

\begin{tabular}{|c|c|c|c|c|c|c|c|c|}
\hline \multirow{2}{*}{$\begin{array}{l}\text { Population microbienne } \\
\text { heure/repas }\end{array}$} & \multicolumn{2}{|c|}{$L A B$} & \multicolumn{2}{|c|}{$L A P$} & \multicolumn{2}{|c|}{$S A B$} & \multicolumn{2}{|c|}{ Effet } \\
\hline & $-1 h$ & $+3 h$ & $-1 h$ & $+3 h$ & $-1 h$ & $+3 h$ & Pop & popxtps \\
\hline \multicolumn{9}{|l|}{ Glycosidases } \\
\hline B-D-Galactosidase & $84,7^{\mathrm{a}}$ & $107,6^{a}$ & $9,5^{\mathrm{a}}$ & $22,1^{a}$ & $169,9^{a}$ & $162,5^{a}$ & * & NS \\
\hline B-D-Xylosidase & $58,9^{a}$ & $91,2 b$ & $22,3^{a}$ & $24,4^{a}$ & $386,6^{a}$ & $289,5^{b}$ & * & $*$ \\
\hline$\beta$-D-Glucosidase & $84,1^{a}$ & $182,6^{b}$ & $217,8^{a}$ & $165,2^{b}$ & $357,1^{a}$ & $340,8^{a}$ & * & * \\
\hline$\alpha$-D-Glucosidase & $32,1^{a}$ & $59,4^{b}$ & $7,5^{\mathrm{a}}$ & $5,9^{a}$ & $59,2^{a}$ & $43,3^{b}$ & * & $\star$ \\
\hline B-D-Cellobiosidase & $22,1^{a}$ & $31,7^{\mathrm{a}}$ & $32,7^{a}$ & $37,3^{a}$ & $135,8^{a}$ & $122,7^{a}$ & * & NS \\
\hline \multicolumn{9}{|l|}{ Polysaccharidases } \\
\hline Carboxyméthylcellulase & $0,3^{a}$ & $0,1^{\mathrm{a}}$ & $3,7^{a}$ & $3,0^{\mathrm{a}}$ & $15,4^{\mathrm{a}}$ & $15,4 a$ & * & NS \\
\hline Xylanase & $2,6^{a}$ & $3,6^{a}$ & $5,6^{a}$ & $2,7^{a}$ & $77,5^{\mathrm{a}}$ & $87,5^{\mathrm{a}}$ & * & NS \\
\hline
\end{tabular}

\footnotetext{
1 Exprimée en nmol de p-nitrophénol libérées/mg de protéines/min (glycosidases) et en $\mathrm{mg}$ de sucres réducteurs libérés/mg de protéines/min (polysaccharidases). " Différence significative 1\%: NS: non significatif. Une lettre différente en exposant correspond à une difference significative entre les temps $-1 \mathrm{~h}$ et $+3 \mathrm{~h}$ pour une même population microbienne.
} 